\title{
Expansive mappings on bounded sets and their application to rational integral equations
}

\author{
Nicolae-Adrian Secelean ${ }^{1} \cdot$ Dariusz Wardowski ${ }^{2}$ \\ Received: 4 October 2019 / Accepted: 28 April 2020 / Published online: 21 May 2020 \\ (c) The Author(s) 2020
}

\begin{abstract}
The aim of this paper is to introduce a new condition of expansiveness which extends the class of the known expansive-type mappings in the domain of closed and bounded subsets of a metric space. For the sum of an expansive mapping in a proposed sense defined on a closed convex and bounded subset of a Banach space and a continuous and compact operator, there are proved some fixed point theorems. There are also provided some examples which motivate our research. Moreover, an application to the existence problem of periodic solutions of nonlinear integral equations with a rational part is provided.
\end{abstract}

Keywords Expansive mapping $\cdot$ Fixed point $\cdot$ Compact mapping $\cdot$ Nonlinear integral equation

Mathematics Subject Classification Primary 47H10 • 47N20

\section{Introduction and preliminaries}

The fixed point tools gain their value through numerous applications in many branches of mathematics. It is recommended that the appropriate assumptions and conditions put on the space and the mappings are natural and possible to meet in typical situations. One of such tools is a well known Krasnosel'skii fixed point theorem, which is an ingenious combination of two fundamental results from the fixed point theory, Schauder's fixed point theorem with a topological nature and Banach contraction principle in a well known metric context. We can cite this theorem as follows:

Dariusz Wardowski

dariusz.wardowski@wmii.uni.lodz.pl

Nicolae-Adrian Secelean

nicolae.secelean@ulbsibiu.ro

1 Department of Mathematics and Computer Science, Lucian Blaga University of Sibiu, Sibiu, Romania

2 Department of Nonlinear Analysis, Faculty of Mathematics and Computer Science, University of Łódź, Łódź, Poland 
Theorem 1.1 [10] If $M$ is a nonempty closed convex and bounded subset of a Banach space $E, A: M \rightarrow E$ is a contraction, $B: M \rightarrow E$ is a continuous compact mapping (i.e. maps a subset of $M$ into a compact subset of $E$ ) and $A(M)+B(M) \subset M$, then $A+B$ admits a fixed point.

In the literature one can find many articles having relations with Theorem 1.1. Burton in [1] obtained a fixed point result for the sum of continuous and compact operator with the so called large contraction. In [7] the authors investigated the case when the mapping $A$ is not a contraction and even $I-A$ need not be injective. There are also many contributions where the quite restrictive and difficult to apply the inclusion $A(M)+B(M) \subset M$ is weakened. E.g. in [2] Burton replaced this condition by the following: if $A x+B y=x$ for some $y \in M$, then $x \in M$. In [12,15], by applying the measure of noncompactness and condensing mappings, the authors obtained the existence of fixed point using the condition $(A+B)(M) \subset M$, which is more flexible in applications. Also, a deep study of Krasnosel'skii type results was presented in [19], where Xiang and Yuan formulated some theorems in a background of strong topology.

It is also worthy to mention here the articles where a contraction mapping is replaced by an expansive one. The examples of contributions where fixed point results for expansive type mappings are investigated may be found in $[5,8,9,11,14,16,17]$. For our purposes, the important results in this context are due to Xiang and Yuan [18]. In their work, the authors studied, for the given metric space $(X, \mathrm{~d})$ and a subset $K \subset X$, the expansive mappings $T: K \rightarrow X$ described by the following inequality:

$$
\mathrm{d}(T x, T y) \geq h \mathrm{~d}(x, y) \text { for all } x, y \in K \text { and some } h>1 .
$$

For the sum of a mapping satisfying the condition (1) and a compact mapping, the authors obtained some fixed point results. For more details we refer the reader to [18].

For our purposes, in the present work we will use a new type of expansive mappings $T: K \rightarrow X$, i.e. satisfying the following condition:

(A) $T$ is injective and there exists $H>0$ such that, for all $x, y \in K, x \neq y$, the following holds:

$$
H \leq \frac{1}{\mathrm{~d}(x, y)}-\frac{1}{\mathrm{~d}(T x, T y)} .
$$

We will show that in a domain of the bounded subsets of the given normed space the condition (A) describes the family of mappings essentially broader than the class of expansive mappings satisfying (1). We will also prove some new fixed point theorems of Krasnosel'skii type. Finally, one of the presented result will be applied to the proof of the existence of a periodic solution of some integral equation. In order to obtain our claims we will also exploit the following result which is a straightforward consequence of Theorem 2.1 in [15].

Corollary 1.1 Let $(X, \mathrm{~d})$ be a complete metric space, $\tau>0$ and let $T: X \rightarrow X$ satisfy:

$$
\mathrm{d}(T x, T y) \leq \frac{\mathrm{d}(x, y)}{1+\tau \mathrm{d}(x, y)} \text { for all } x, y \in X .
$$

Then $T$ has a unique fixed point.

Proof It is enough to take in [15, Th. 2.1] the function $F(t)=-1 / t$ and $\varphi(t)=\tau$.

For the convenience of the reader we also present the following version of Schauder's theorem which can be found e.g. in [4]. The original statement of this theorem can be found in [13]. 
Theorem 1.2 [4] Let $K$ be a nonempty closed convex subset of a Banach space E. Suppose that the mapping $S: K \rightarrow K$ is continuous and $S(K)$ resides in a compact subset of $E$. Then $S$ has at least one fixed point in $K$.

\section{Results}

We start with a simple but important observation which is a consequence of Corollary 1.1.

Theorem 2.1 Let $K$ be a nonempty closed subset of a complete metric space $(X, \mathrm{~d})$. If a mapping $T: K \rightarrow X$ satisfies $(A)$ and $K \subset T(K)$ then $T$ has a unique fixed point.

Proof (A) implies that the inverse $T^{-1}: T(K) \rightarrow K$ of the mapping $T$ exists. Take $u, v \in$ $T(K)$ such that $u \neq v$. One can find $x, y \in K, x \neq y$ for which $T x=u, T y=v$. Also, by (A), there exits $H>0$ such that:

$$
H \leq \frac{1}{\mathrm{~d}(x, y)}-\frac{1}{\mathrm{~d}(T x, T y)}=\frac{1}{\mathrm{~d}\left(T^{-1} u, T^{-1} v\right)}-\frac{1}{\mathrm{~d}(u, v)} .
$$

In consequence, and due to the fact that $K \subset T(K)$, we obtain:

$$
\mathrm{d}\left(T^{-1} u, T^{-1} v\right) \leq \frac{\mathrm{d}(u, v)}{1+H \mathrm{~d}(u, v)} \text { for all } u, v \in K .
$$

From the closedness of $K$ we can apply Corollary 1.1 which yields the existence of a unique $\bar{x} \in K$ such that $T^{-1} \bar{x}=\bar{x}$ and hence $T \bar{x}=\bar{x}$.

Example 2.1 (a) Let $a>1$ and consider $X:=[0, a]$ with a standard metric, $1<\lambda \leq a$, $K:=[0,1], T x:=\lambda x, x \in K$. Then $T$ satisfies (A) with $H:=(\lambda-1) / \lambda$ and $K \subset T(K)$.

(b) Consider the Banach space $X:=C(I)$ of all real-valued continuous functions defined on the closed and bounded interval $I$ with maximum norm and a subset $K$ of $X$ of the form

$$
K:=\{x \in X: x \geq 0,\|x\| \leq \lambda\}, \text { where } 0<\lambda<1 .
$$

Define the mapping $T: K \rightarrow X$ by

$$
(T x)(t):=\frac{x(t)}{1-x(t)}, t \in I, x \in K .
$$

We prove that $T$ satisfies the condition (A) for every $H \in(0,1]$. For this purpose, let $x, y \in K, x \neq y$. One can choose $t \in I$ such that $\|x-y\|=|x(t)-y(t)|>0$. We have

$$
\frac{1}{|x(t)-y(t)|}-\frac{1}{|(T x)(t)-(T y)(t)|}=\frac{x(t)-x(t) y(t)+y(t)}{|x(t)-y(t)|} .
$$

Since it is obvious that $x(t)-x(t) y(t)+y(t) \geq|x(t)-y(t)|$, from the above we obtain

$$
\frac{1}{|x(t)-y(t)|}-\frac{1}{|(T x)(t)-(T y)(t)|} \geq 1
$$

that is

$$
\frac{1}{\|x-y\|} \geq \frac{1}{|(T x)(t)-(T y)(t)|}+1 \geq \frac{1}{\|T x-T y\|}+1 .
$$


Consequently, the inequality in (A) is satisfied for all $H \in(0,1]$. Note that $T$ is an injective mapping on $K$ and $K \subset T(K)$. Thus all the assumptions of Theorem 2.1 are satisfied.

Moreover, observe that

$$
\inf _{a, b \in[0, \lambda], a \neq b} \frac{|T a-T b|}{|a-b|}=\inf _{a, b \in[0, \lambda], a \neq b} \frac{1}{(1-a)(1-b)}=1,
$$

therefore there is no $h>1$ such that $\|T x-T y\| \geq h\|x-y\|$ for all $x, y \in K$. In consequence the mapping $T$ is not the one of the expansive mappings which were studied in [18] and the results presented there are not applicable.

Also observe that in this case, for the mapping $T^{-1}: T(K) \rightarrow K$ as a contractive one, i.e. $\left\|T^{-1} x-T^{-1} y\right\|<\|x-y\|$, for $x \neq y$, we cannot apply the known fixed point results (e.g. Edelstein [3]) since $T(K)$ is not compact.

In the following we highlight a necessary property of $K$ which is a consequence of the condition (A). We also present a relation between (A) and (1).

Remark 2.1 Let $(X, \mathrm{~d})$ be a metric space and $\varnothing \neq K \subset X$.

$1^{\circ}$. If $T: K \rightarrow X$ fulfils the condition (A), then $K$ is bounded.

$2^{\circ}$. If $K$ is bounded and $T: K \rightarrow X$ is expansive in the sense (1), then $T$ satisfies (A).

Proof $1^{\circ}$. If $K$ is unbounded, then $\sup _{x, y \in K} \mathrm{~d}(x, y)=\infty$ so there are $\left(x_{n}\right),\left(y_{n}\right) \subset K$, $x_{n} \neq y_{n}$ such that $\lim _{n} \mathrm{~d}\left(x_{n}, y_{n}\right)=\infty$, that is $\lim _{n} \frac{1}{\mathrm{~d}\left(x_{n}, y_{n}\right)}=0$. Since $T$ is injective, $T x_{n} \neq T y_{n}$ for all $n$ hence, by (A), one has

$$
H<\frac{1}{\mathrm{~d}\left(T x_{n}, T y_{n}\right)}+H \leq \frac{1}{\mathrm{~d}\left(x_{n}, y_{n}\right)} \underset{n}{\longrightarrow} 0, \forall n
$$

which contradicts the positivity of $H$.

$2^{\circ}$. Let $M:=\sup _{x, y \in K} \mathrm{~d}(x, y), M \in(0, \infty)$ and $h>1$ be such that $\mathrm{d}(T x, T y) \geq$ $h \mathrm{~d}(x, y)$, for all $x, y \in K$. Set $H \in\left(0, \frac{1}{M}-\frac{1}{h M}\right)$. Choose $x, y \in K, x \neq y$. We have $1-H \mathrm{~d}(x, y) \geq 1-H M>0$ and $h \geq \frac{1}{1-H \mathrm{~d}(x, y)}$ so $h \mathrm{~d}(x, y) \geq \frac{\mathrm{d}(x, y)}{1-H \mathrm{~d}(x, y)}$. Using now the expansiveness of $T$, one has

$$
\mathrm{d}(T x, T y) \geq \frac{\mathrm{d}(x, y)}{1-H \mathrm{~d}(x, y)},
$$

which is exactly the inequality in (A).

One can easy observe that, if the set $K$ is unbounded, the expansiveness (1) does not imply (A) (e.g $K=\mathbb{R}$ with standard metric and $T x=h x, h>1$ ). Also, in view of Example 2.1 (b), in bounded sets (in particular on a compact subset of a metric space) expansiveness described by (A) is essentially more general from (1). This is essential for our existence problem considered in the last section of the article.

The following theorem states that the operator $I-T$ with $T$ satisfying the condition (A) is invertible.

Proposition 2.1 Consider a normed space $(X,\|\cdot\|), \varnothing \neq K \subset X$ and let $T: K \rightarrow X$ satisfy (A). Then the inverse $(I-T)^{-1}:(I-T)(K) \rightarrow K$ exists and is continuous.

Proof Take $x, y \in K, x \neq y$ and denote $F:=I-T$. By (A) we get

$$
1-H\|x-y\| \geq \frac{\|x-y\|}{\|T x-T y\|}>0 \text { for some } H>0 .
$$


We also easily have

$$
0<\|T x-T y\| \leq\|F x-F y\|+\|x-y\| .
$$

(2) and (3) imply

$$
\frac{1}{\|F x-F y\|+\|x-y\|} \leq \frac{1}{\|T x-T y\|} \leq \frac{1-H\|x-y\|}{\|x-y\|} .
$$

From the above and (2) we obtain

$$
\begin{aligned}
\|F x-F y\| & \geq \frac{\|x-y\|}{1-H\|x-y\|}-\|x-y\| \\
& =\frac{H\|x-y\|^{2}}{1-H\|x-y\|} .
\end{aligned}
$$

Therefore for all $x, y \in K$ the following inequality holds

$$
\|F x-F y\| \geq H\|x-y\|^{2} .
$$

Inequality (4) implies that $F$ is injective assuring that $F$ is invertible. Moreover, also from (4), for every $x, y \in(I-T)(K)$ we see that

$$
\left\|F^{-1} x-F^{-1} y\right\|^{2} \leq \frac{1}{H}\|x-y\|
$$

which finally gives the continuity of $F^{-1}$.

Theorem 2.2 Let $K$ be a nonempty closed convex and bounded subset of a Banach space $E$ and let $T, S: K \rightarrow E$ be the mappings satisfying the following conditions:

(i) $S$ is continuous and $S(K)$ resides in a compact subset of $E$,

(ii) $T$ satisfies $(A)$,

(iii) for every $z \in S(K)$ the inclusion $K \subset z+T(K)$ holds.

Then there exists $\bar{x} \in K$ such that $S \bar{x}+T \bar{x}=\bar{x}$.

Proof First, observe that for every $z \in S(K)$ the mapping $T+z$, due to (ii) and (iii), satisfies the assumptions of Theorem 2.1. Hence the equation

$$
T x+z=x
$$

has only one solution $x \in K$. Denote by $v$ a function which for every $z \in S(K)$ assigns $x \in K$ such that (5) holds. Therefore for every $z \in S(K)$ we have:

$$
T(v(z))+z=v(z) .
$$

Consider $z_{1}, z_{2} \in S(K)$. Without reducing the generality of our considerations we can assume that $v\left(z_{1}\right) \neq v\left(z_{2}\right)$. Since $T$ satisfies (A), one can find $H>0$ such that

$$
H \leq \frac{1}{\left\|v\left(z_{1}\right)-v\left(z_{2}\right)\right\|}-\frac{1}{\left\|T\left(v\left(z_{1}\right)\right)-T\left(v\left(z_{2}\right)\right)\right\|} .
$$

By the above we have the following inequality:

$$
\left\|v\left(z_{1}\right)-v\left(z_{2}\right)\right\| \leq \frac{\left\|T\left(v\left(z_{1}\right)\right)-T\left(v\left(z_{2}\right)\right)\right\|}{1+H\left\|T\left(v\left(z_{1}\right)\right)-T\left(v\left(z_{2}\right)\right)\right\|} .
$$

Moreover, by (6) the following holds:

$$
\left\|T\left(v\left(z_{1}\right)\right)-T\left(v\left(z_{2}\right)\right)\right\| \leq\left\|v\left(z_{1}\right)-v\left(z_{2}\right)\right\|+\left\|z_{1}-z_{2}\right\| .
$$


From (7), (8) and the monotonicity of the function $[0, \infty) \ni t \mapsto t /(1+H t)$ we obtain:

$$
\begin{aligned}
\left\|v\left(z_{1}\right)-v\left(z_{2}\right)\right\| \leq & \frac{\left\|v\left(z_{1}\right)-v\left(z_{2}\right)\right\|+\left\|z_{1}-z_{2}\right\|}{1+H\left\|v\left(z_{1}\right)-v\left(z_{2}\right)\right\|+H\left\|z_{1}-z_{2}\right\|} \\
= & \frac{\left\|v\left(z_{1}\right)-v\left(z_{2}\right)\right\|}{1+H\left\|v\left(z_{1}\right)-v\left(z_{2}\right)\right\|+H\left\|z_{1}-z_{2}\right\|} \\
& +\frac{\left\|z_{1}-z_{2}\right\|}{1+H\left\|v\left(z_{1}\right)-v\left(z_{2}\right)\right\|+H\left\|z_{1}-z_{2}\right\|} \\
\leq & \frac{\left\|v\left(z_{1}\right)-v\left(z_{2}\right)\right\|}{1+H\left\|v\left(z_{1}\right)-v\left(z_{2}\right)\right\|}+\frac{\left\|z_{1}-z_{2}\right\|}{1+H\left\|z_{1}-z_{2}\right\|} .
\end{aligned}
$$

In consequence, we have:

$$
\frac{H\left\|v\left(z_{1}\right)-v\left(z_{2}\right)\right\|^{2}}{1+H\left\|v\left(z_{1}\right)-v\left(z_{2}\right)\right\|} \leq \frac{\left\|z_{1}-z_{2}\right\|}{1+H\left\|z_{1}-z_{2}\right\|},
$$

which yields that $\left\|z_{1}-z_{2}\right\| \rightarrow 0$ implies $\left\|v\left(z_{1}\right)-v\left(z_{2}\right)\right\| \rightarrow 0$ and thus $v$ is continuous on $S(K)$. From the continuity of $S$ it follows the continuity of the mapping $v S: K \rightarrow K$ and also that $(v S)(K)$ resides in a compact subset of $E$. According to Theorem 1.2 there exists $\bar{x} \in K$ such that $v(S(\bar{x}))=\bar{x}$. From (6) we get

$$
T(v(S(\bar{x})))+S(\bar{x})=v(S(\bar{x}))
$$

what finally gives $T \bar{x}+S \bar{x}=\bar{x}$.

\section{Nonlinear integral equation with delay}

Consider the following nonlinear integral equation with delay of rational type.

$$
x(t)=\frac{p(t) x(t-\tau)}{1-q(t) x(t-\tau)}+\int_{-\infty}^{t} f(t-s) g(x(s)) d s,
$$

where $\tau>0$ is fixed, the mappings $p, q: \mathbb{R} \rightarrow \mathbb{R}$ are continuous and periodic with period $P>0, f, g: \mathbb{R} \rightarrow \mathbb{R}$ are continuous. We assume that:

(C1) $p(t) \geq(q(t)+1)^{2}$ for all $t \in \mathbb{R}$,

(C2) $q_{m}:=\min _{t \in[0, P]} q(t)>0, q_{M}:=\max _{t \in[0, P]} q(t)<1$,

(C3) $M:=\sup _{t \in \mathbb{R}} \int_{-\infty}^{t}|f(t-s)| d s<\infty$ and $\int_{-\infty}^{t}\left|f^{\prime}(t-s)\right| d s<\infty$ for all $t \in \mathbb{R}$,

$$
M \max _{|t| \leq 1} g(t) \leq \frac{p_{M}+q_{M}-1}{1-q_{M}},
$$

where $p_{M}:=\max _{t \in[0, P]} p(t)$ and $M$ is from (C3).

In order to solve the existence problem for the Eq. (9) we will apply Theorem 2.2.

Consider the Banach space $E$ of all continuous periodic self-mappings on $\mathbb{R}$ with period $P>0$, where for $x \in E$ we denote by $\|x\|$ the supremum norm, i.e. $\|x\|=\sup _{t \in[0, P]}|x(t)|$. Let $K$ be a subset of $E$ of the form

$$
K:=B_{1}:=\{x \in E:\|x\| \leq 1\} .
$$

Theorem 3.1 If the conditions (C1)-(C4) are satisfied then the Eq. (9) has a P-periodic solution. 
Proof Let us put

$$
(T x)(t):=\frac{p(t) x(t-\tau)}{1-q(t) x(t-\tau)}, \quad(S x)(t):=\int_{-\infty}^{t} f(t-s) g(x(s)) d s, \quad x \in K .
$$

Observe that, by (C2), $1-q(t) x(t-\tau) \neq 0$ for all $t \in \mathbb{R}$ since $q(t)<1$ and $x \in K$. The problem (9) reduces to finding a fixed point of the equation $x=T x+S x$. Consider $x, y \in K$ such that $T x \neq T y$. For $t \in[0, P]$ we have

$$
\begin{aligned}
& \frac{1}{|x(t)-y(t)|}-\frac{1}{|(T x)(t+\tau)-(T y)(t+\tau)|} \\
& \quad=\frac{1}{|x(t)-y(t)|}-\frac{(1-q(t+\tau) x(t))(1-q(t+\tau) y(t))}{p(t+\tau)|x(t)-y(t)|} \\
& \quad=\frac{q(t+\tau)}{p(t+\tau)} \cdot \frac{(p(t+\tau)-1) / q(t+\tau)+x(t)+y(t)-q(t+\tau) x(t) y(t)}{|x(t)-y(t)|} .
\end{aligned}
$$

By (C1), we obtain

$$
\begin{aligned}
& \frac{1}{|x(t)-y(t)|}-\frac{1}{|(T x)(t+\tau)-(T y)(t+\tau)|} \\
& \quad \geq \frac{q(t+\tau)}{p(t+\tau)} \cdot \frac{q(t+\tau)+2+x(t)+y(t)-q(t+\tau) x(t) y(t)}{|x(t)-y(t)|} \\
& \quad \geq \frac{q_{m}}{p_{M}} \cdot \frac{2+x(t)+y(t)}{|x(t)-y(t)|} \\
& \quad \geq \frac{q_{m}}{p_{M}} .
\end{aligned}
$$

From the above inequalities it follows

$$
\begin{aligned}
\frac{\|T x-T y\|}{1+\frac{q_{m}}{p_{M}}\|T x-T y\|} & \geq \frac{|(T x)(t+\tau)-(T y)(t+\tau)|}{1+\frac{q_{m}}{p_{M}}|(T x)(t+\tau)-(T y)(t+\tau)|} \\
& \geq|x(t)-y(t)|
\end{aligned}
$$

for all $t \in[0, P]$. In consequence, we get

$$
\frac{\|T x-T y\|}{1+\frac{q_{m}}{p_{M}}\|T x-T y\|} \geq\|x-y\| .
$$

Moreover, observe that $T$ is an injective mapping on $K$. Thus (A) is satisfied for $H=\frac{q_{m}}{p_{M}}$.

Using similar arguments like in [18] one can prove that the mapping $S$ is completely continuous (i.e. it is continuous and the image $S(K)$ is contained in a compact subset of $E$ ) and hence the condition (i) of Theorem 2.2 is satisfied.

We now consider $z \in S(K)$. There exists $w \in K$ such that $z(t)=(S w)(t)$ for all $t \in[0, P]$. In order to show the inclusion (iii) in Theorem 2.2 take $x \in K$. By (C4) we have

$$
\|x-z\| \leq\|x\|+\|S w\| \leq 1+M \max _{|t| \leq 1} g(t) \leq \frac{p_{M}}{1-q_{M}} .
$$

Moreover, notice that

$$
T(K)=B_{r}, \text { where } r:=\frac{p_{M}}{1-q_{M}} .
$$

Indeed, first observe that

$$
\|T w\| \leq r \text { for every } w \in K \text {. }
$$


On the other hand taking any $y \in E$ such that $\|y\| \leq r$ one can put

$$
w(t)=\frac{y(t+\tau)}{p(t+\tau)+q(t+\tau) y(t+\tau)} .
$$

We see that $w \in K$ and $T w=y$. From (10) and (11) we finally have $x-z \in T(K)$, i.e. the condition (iii) is satisfied and the proof is completed.

Remark 3.1 Note that the thesis enunciated in Theorem 3.1 cannot be obtained by means of expansive mappings considered in [18]. Indeed, observe that the operator

$$
(T x)(t)=\frac{p(t) x(t-\tau)}{1-q(t) x(t-\tau)}
$$

used in the proof of the theorem in general is not an expansive mapping described by the inequality (1). Indeed, if we consider e.g. $q(t):=q_{0} \in(0,1)$ for all $t \in \mathbb{R}, p(t):=p_{0}:=$ $\left(q_{0}+1\right)^{2}$ for all $t \in \mathbb{R}$ then for all constant functions $x, y \in K, x \neq y$ we get:

$$
|T x-T y|=\frac{p_{0}|x-y|}{\left(1-q_{0} x\right)\left(1-q_{0} y\right)} .
$$

Therefore

$$
\inf _{x, y \in[-1,1]} \frac{|T x-T y|}{|x-y|}=\inf _{x, y \in[-1,1]} \frac{p_{0}}{\left(1-q_{0} x\right)\left(1-q_{0} y\right)}=\frac{p_{0}}{\left(1+q_{0}\right)\left(1+q_{0}\right)}=1 .
$$

In consequence, one cannot find $h>1$ satisfying (1).

Acknowledgements University of Lodz. The authors want to express their gratitude to the reviewers for valuable comments that contributed to the final shape of the article. N.-A. Secelean is financed by Lucian Blaga University of Sibiu \& Hasso Plattner Foundation research grants LBUS-IRG-2019-05.

Open Access This article is licensed under a Creative Commons Attribution 4.0 International License, which permits use, sharing, adaptation, distribution and reproduction in any medium or format, as long as you give appropriate credit to the original author(s) and the source, provide a link to the Creative Commons licence, and indicate if changes were made. The images or other third party material in this article are included in the article's Creative Commons licence, unless indicated otherwise in a credit line to the material. If material is not included in the article's Creative Commons licence and your intended use is not permitted by statutory regulation or exceeds the permitted use, you will need to obtain permission directly from the copyright holder. To view a copy of this licence, visit http://creativecommons.org/licenses/by/4.0/.

\section{References}

1. Burton, T.A.: Integral equations, implicit functions and fixed points. Proc. Am. Math. Soc. 124, 2383-2390 (1996)

2. Burton, T.A.: A fixed-point theorem of Krasnoselskii. Appl. Math. Lett. 11(1), 85-88 (1998)

3. Edelstein, M.: On fixed and periodic points under contractive mappings. J. Lond. Math. Soc. 37, 74-79 (1962)

4. Gasiński, L., Papageorgiu, N.S.: Nonlinear Analysis, vol. 9. Chapman and Hall/CRC, London (2006)

5. Górnicki, J.: Fixed point theorems for $F$-expanding mappings. Fixed Point Theory Appl. 2017, 9 (2017). https://doi.org/10.1186/s13663-017-0602-3

6. Istratescu, V.I.: Fixed Point Theory. D. Reidel Publishing Company, Boston (1981)

7. Liu, Y., Li, Z.: Krasnoselskii type fixed point theorems and applications. Proc. Am. Math. Soc. 136, 1213-1220 (2008)

8. Kang, S.M.: Fixed point for expansion mappings. Math. Japn. 38, 713-717 (1993)

9. Kang, S.M., Khan, M.S., Sessa, S.: Some theorems on expansion mappings and their fixed points. Demonstr. Math. 19, 673-683 (1986) 
10. Krasnosel'skii, M.A.: Some problems of nonlinear analysis. Am. Math. Soc. Transl. Ser. 2(10), 345-409 (1958)

11. Park, S., Rhoades, B.E.: Some fixed point theorems for expansion mappings. Math. Jpn. 33, 129-132 (1988)

12. Przeradzki, B.: A generalization of Krasnosel'skii fixed point theorem for sums of compact and contractible maps with application. Cent. Eur. J. Math. 10, 2012-2018 (2012)

13. Schauder, J.: Der Fixpunktsatz in funktionalräumen. Stud. Math. 2, 171-180 (1930)

14. Wang, S.Z., Li, B.Y., Gao, Z.M.: Some fixed point theorems on expansion mappings. Adv. Math. (China) 2, 149-153 (1982)

15. Wardowski, D.: Solving existence problems via $F$-contractions. Proc. Am. Math. Soc. 146, 1585-1598 (2018)

16. Wang, F., Wang, F.: Krasnosel'skii type fixed point theorem for nonlinear expansion. Fixed Point Theory 13(1), 285-291 (2012)

17. Xiang, T.: Notes on expansive mappings and a partial answer to Nirenberg's problem. Electron. J. Differ. Equ. 02, 1-16 (2013)

18. Xiang, T., Yuan, R.: A class of expansive-type Krasnosel'skii fixed point theorems. Nonlinear Anal. 71, 3229-3239 (2009)

19. Xiang, T., Yuan, R.: A note on Krasnosel'skii fixed point theorem. Fixed Point Theory Appl. 99, 1-8 (2015)

Publisher's Note Springer Nature remains neutral with regard to jurisdictional claims in published maps and institutional affiliations. 